\title{
Economic MPC for the Management of Drinking Water Networks
}

\author{
J.M. Grosso ${ }^{1}$, C. Ocampo-Martínez ${ }^{1}$, Senior Member, IEEE, V. Puig ${ }^{1}$, D. Limon ${ }^{2}$ and M. Pereira ${ }^{2}$
}

\begin{abstract}
This paper addresses the management of drinking water networks (DWNs) regarding a multi-objective cost function by means of economically-oriented model predictive control (EMPC) strategies. Specifically, assuming the water demand and the energy price as periodically time-varying signals, this paper shows that the EMPC framework is flexible to enhance the control of DWNs without relying on hierarchical control schemes that require the use of real-time optimisers (RTO) or steady-state target optimisers (SSTO) in an upper layer. Four different MPC strategies are discussed in this paper: a hierarchical two-layer approach, a standard EMPC where the multi-objective cost function is optimised directly, and two different modifications of the latter, which are meant to overcome possible feasibility losses in the presence of changing operating patterns. The discussed schemes are tested and compared by means of a case study taken from a part of the Barcelona DWN.
\end{abstract}

\section{INTRODUCTION}

Drinking Water Networks (DWNs) form the link between urban water supply systems and drinking water consumption nodes. These networks are vital for the normal functioning of modern society and maintaining a truly sustainable service is a must in these systems. The management of DWNs involves optimising systematically and simultaneously a collection of (generally conflicting) heterogeneous performance criteria while being subject to different control specifications, constraints and disturbances, which are affected by the dynamism of the economic markets, the social behaviour and the local/regional legislations.

The complexity of DWNs, (i.e., dimensionality, nonlinearities, infrastructure constraints, uncertainties), the stronger requirements for water transport service quality and the need for a sustainable exploitation of the available resources make the management of these systems a challenging control problem that has caught the attention of the scientific community, see e.g., [2]-[5], [10], [16], [18]. As discussed in the aforementioned references, several approaches are reported in the literature to address the operation of DWNs, ranging from heuristics and expert systems to more advanced mathematical modelling and optimisation techniques, hierarchical-decompositions, combinatorial

*This work has been partially funded by the EU Project EFFINET (FP7ICT-2011-8-31855) and the DGR of Generalitat de Catalunya (SAC group Ref. 2009/SGR/1491)

${ }^{1}$ J. M. Grosso, C. Ocampo-Martinez and V. Puig are with the Advanced Control Systems Research Group (SAC), at the Institut de Robòtica i Informàtica Industrial (CSIC-UPC). Llorens i Artigas 4-6, 08028. Barcelona, Spain. E-mail: \{jgrosso, cocampo, vpuig\}@iri.upc.edu.

${ }^{2} \mathrm{D}$. Limon and M. Pereira are with the Departamento de Ingeniería de Sistemas y Automática, Universidad de Sevilla, Escuela Superior de Ingenieros, Camino de los Descubrimientos s/n 41092, Sevilla, Spain. Email: $\{d l m$, mpereiram $\} @ u s . e s$ schemes, and more recently, model predictive control (MPC). This latter one has proven to be one of the most effective and accepted control strategies for large-scale complex systems due to its flexibility to manage constraints and to optimise multi-objective problems as the ones encountered in the management of water systems [13]. The basic idea of MPC is to exploit a model of the network to simulate its future evolution over a prediction horizon and compute an optimal control action (with respect to a predefined cost function) by solving at each time instant an open-loop optimisation problem in a receding horizon fashion [12].

Within the active research on MPC strategies for economic operation of systems, the predominant approach is to consider a hierarchical control structure [17], where standard MPC controllers are designed for tracking operational setpoints that are computed usually in an upper layer (i.e., RTO, SSTO) with complex non-linear stationary models and with larger sampling times than the regulatory MPC layer. Nevertheless, as discussed in [7], model inconsistencies, setpoint changes, time-varying parameters, disturbances, and time-scale differences may lead the system to unreachable set-points, suboptimal economic performance and feasibility loss. In order to tackle some of the main drawbacks of the typical hierarchical scheme and take more economic profit from the transitory behaviour of the system, some authors have proposed to integrate the economic optimisation within the MPC using either a two-layer approach, see, e.g., [6], [19], or a single-layer approach, i.e., the so called economic MPC (EMPC) [15]. It has been shown in the aforementioned references that closed-loop stability and/or average asymptotic performance can be guaranteed under some form of dissipativity, duality or convexity assumptions. Nevertheless, as shown in [11], the feasibility of the overall problem might be lost if the economic criteria changes (either under the mentioned one-layer or two-layer schemes), therefore, modified single-layer based controllers that inherit the feasibility guarantee of tracking MPC and the optimality of EMPC have been proposed in this latter reference.

Recent studies on control of DWNs are focused on the design of MPC controllers that directly optimise a (nonstandard) economic cost function, see e.g., [13], [14], not to obtain steady-state set-points but target trajectories for low-level PID controllers. This latter is the case of interest of this paper, where a stationary operation is not beneficial and possible periodicity of time-varying parameters can be exploited for economic profitability. Despite the good practical performance reported in the aforementioned references, stability and feasibility conditions were not considered.

The main contribution of this paper is the comparison 
of several economically-oriented MPC schemes applied to the management of DWNs. Moreover, assuming the water demand and the energy price as periodically time-varying signals, this paper uses the results in [9], [11], [15], [20], and adds periodic terminal constraints to the MPC controller developed in [14] to equip it with guarantees of recursive feasibility and convergence. Even when this paper uses a small-scale case study, the proof of concept may be potentially extended to large-scale DWNs.

The paper is organized as follows. Section II describes a control-oriented flow-based model for DWNs and states the control objective. Section III formulates the MPC strategies to be compared for the economic management of DWN. Section IV describes the case study and presents the results of applying four EMPC schemes. Section V highlights the concluding remarks that can be drawn from the results presented in this paper as well as some future research directions.

\section{PROBLEM STATEMENT}

\section{A. Control-Oriented Modelling}

This paper considers a general DWN being represented by a directed graph $G(\mathcal{V}, \mathcal{E})$, where a set of elements, i.e., $n_{s}$ sources, $n_{x}$ storage tanks, $n_{q}$ intersection nodes, and $n_{d}$ sinks, are represented by $v \in \mathcal{V}$ vertices, which are connected by $a \in \mathcal{E}$ directed links. Due to the network function, water is transported along the links by $n_{u}$ flow actuators (i.e., pipes and valves), passing through reservoirs or tanks, from specific origin locations to specific destination locations. The network is subject to several capacity and operational constraints, and to measured stochastic flows to sinks driven by customers water demand.

Setting the volume in the storage tanks as the state variable $x_{k} \in \mathbb{R}^{n_{x}}$, the flow through the actuators as the manipulated inputs $u_{k} \in \mathbb{R}^{n_{u}}$, and the demanded flow as measured disturbances $d_{k} \in \mathbb{R}^{n_{d}}$, then the nominal controloriented model of the DWN $G(\mathcal{V}, \mathcal{E})$ may be abstracted and described by the following set of linear discrete-time difference-algebraic equations for all time instant $k \in \mathbb{N}$ :

$$
\begin{aligned}
x_{k+1} & =A x_{k}+B u_{k}+B_{d} d_{k}, \\
0 & =E_{u} u_{k}+E_{d} d_{k},
\end{aligned}
$$

where the difference equations in (1a) describe the dynamics of the storage tanks, and the algebraic equations in (1b) describe the static relations (i.e., mass balance at junction nodes) in the network. Moreover, $A, B, B_{d}, E_{u}, E_{d}$, are time-invariant matrices of suitable dimensions dictated by the network topology.

The states and control inputs are subject to hard constraints due physical and operational limits, i.e.,

$$
\begin{array}{ll}
x_{\min } \leq x_{k} \leq x_{\max }, & \forall k \in \mathbb{N} \\
u_{\min } \leq u_{k} \leq u_{\max }, & \forall k \in \mathbb{N}
\end{array}
$$

where $x_{\min }$ and $x_{\max }$ are vectors in $\mathbb{R}^{n_{x}}$ of the minimum and maximum volume of water that the tanks are able to store, and similarly, $u_{\min }$ and $u_{\max }$ are vectors in $\mathbb{R}^{n_{u}}$ of the minimum and maximum allowable water flow through actuators. Moreover, for safety and supply service reliability, the states are subject to the following management soft constraint:

$$
x_{k} \geq s-\xi_{k}, \quad \xi_{k} \geq 0, \quad \forall k \in \mathbb{N}
$$

where $s \in \mathbb{R}_{+}^{n_{x}}$ is a positive vector of base stocks (minimal volume in each tank to avoid stock-outs) and $\xi_{k} \in \mathbb{R}_{+}^{n_{x}}$ is a vector of positive slack variables to be minimised, which represents the amount of water volume in tanks that is allowed to go below from the desired base stocks.

Assumption 1: The state $x_{k}$ and the demand $d_{k}$ are measured at any time instant $k$, and the pair $(A, B)$ is controllable. Furthermore, all elements operate with a common review period $\Delta t$ and all storage tanks are subject to the same replenishment policy.

Assumption 2: Water demands are non-stationary and present a cyclic pattern with known period $T \geq 1$, hence, $d_{k+T}=d_{k}$ for all $k \in \mathbb{N}$. The admissible demands lie in a polytopic convex set, i.e., $d_{k} \in \mathbb{W} \subset \mathbb{R}^{n_{d}}$ for all $k \in \mathbb{N}$. The periodic trajectory of this exogenous signal, i.e., $\mathbf{d}_{k}=\left\{d_{i}\right\}_{i=k}^{k+T-1}$, will be assumed as perfectly known when designing the nominal predictive controllers.

\section{B. System Management Criteria}

The management of a DWN is a multi-objective optimisation problem. In this paper, three operational goals with different nature are considered, i.e., economic, safety, and smoothness objectives. Therefore, the control task is to design a control law $u_{k}=\kappa\left(x_{k}, \mathbf{d}_{k}\right)$ that minimises the following cost functions:

$$
\begin{aligned}
& \ell_{k}^{e} \triangleq \alpha_{k}^{\top} W_{e} u_{k}, \\
& \ell_{k}^{s} \triangleq \xi_{k}^{\top} W_{s} \xi_{k}, \\
& \ell_{k}^{\Delta} \triangleq \Delta u_{k}^{\top} W_{\Delta u} \Delta u_{k},
\end{aligned}
$$

where $\ell_{k}^{e} \in \mathbb{R}_{\geq 0}$ represents the economic cost of network operation that depends on a time-of-use pricing scheme given by $\alpha_{k} \triangleq\left(\alpha_{1}+\alpha_{2, k}\right) \in \mathbb{R}_{+}^{n_{u}}$, which takes into account a fixed water production cost $\alpha_{1} \in \mathbb{R}_{+}^{n_{u}}$ and a time-varying water pumping cost $\alpha_{2, k} \in \mathbb{R}_{+}^{n_{u}}$ that changes every time instant $k$ according to the dynamic electric tariff. The cost $\ell_{k}^{s} \in \mathbb{R}_{\geq 0}$ penalises the amount of water volume that goes below from the pre-specified security threshold $s$ in (3), i.e., $\xi_{k}=x_{k}-s$ if $x_{k} \leq s$, otherwise $\xi_{k}=0$. The cost $\ell_{k}^{\Delta} \in \mathbb{R}_{>0}$ represents the penalisation of control signal variations $\Delta u_{k} \triangleq u_{k}-u_{k-1}$, which assures a smooth operation and extends actuators life. Furthermore, $W_{e}, W_{s}$, $W_{\Delta u}$ are diagonal positive definite matrices that weight each decision variable in their corresponding cost function.

Assumption 3: The pricing in the economic cost is assumed to be periodic, i.e., $\alpha_{k}=\alpha_{k+T}$ for all $k \in \mathbb{N}$.

To achieve the control task, the above predefined objectives are aggregated in a weighted sum stage cost function

$$
V_{k}(x, u) \triangleq \lambda_{1} \ell_{k}^{e}+\lambda_{2} \ell_{k}^{s}+\lambda_{3} \ell_{k}^{\Delta}
$$


where $\lambda_{1}, \lambda_{2}, \lambda_{3}$ are positive scalars that allow to prioritise the impact of each involved objective on the overall performance of the system. Ideally, the resultant control strategy should fill the tanks during the periods of lower energy cost with water taken from the cheapest sources, and deplete them when compensating demands. Given Assumptions 2 and 3, the optimal nominal behaviour of the system for a known period $T \geq 1$ is defined by the $T$-periodic sequences $\mathbf{x}^{\star}=\left\{x_{i}^{\star}\right\}_{i=0}^{T-1}$ and $\mathbf{u}^{\star}=\left\{u_{i}^{\star}\right\}_{i=0}^{T-1}$, which result from solving the following finite horizon optimisation problem (FHOP):

$$
J_{p}^{\star} \triangleq \min _{\mathbf{x}^{\star} \mathbf{u}^{\star} \boldsymbol{\xi}^{\star}} \sum_{k=0}^{T-1} \tilde{V}_{k}\left(x_{k}, u_{k}\right)
$$

subject to (1), (2), (3) $\forall k \in \mathbb{N}_{[0, T-1]}$ and

$$
x_{T}=x_{0}
$$

where $\tilde{V}_{k}\left(x_{k}, u_{k}\right) \triangleq V_{k}\left(x_{k}, u_{k}\right)+\delta\left\|x_{k}\right\|^{2}$. This last term, i.e., $\delta\left\|x_{k}\right\|^{2}$ with $\delta \in \mathbb{R}_{+}$and sufficiently small, is a convex regularisation term added to enforce the uniqueness of the solution to (6) given that the cost function (5) is originally not convex with respect to $x$. Defining the weighting matrices $W_{s}$ and $W_{\Delta u}$ in (4) to be positive definite, then $\tilde{V}_{k}\left(x_{k}, u_{k}\right)$ is bounded and strictly convex by design with respect to $(x, u, \xi)$. Hence, (6) has a unique solution for a given nominal periodic sequence of water demand $\mathbf{d}_{k}=\left\{d_{i}\right\}_{i=k}^{k+T-1}$ and economic pricing $\boldsymbol{\alpha}_{k}=\left\{\alpha_{i}\right\}_{i=k}^{k+T-1}$. Therefore, the equality $\left(x_{k}^{\star}, u_{k}^{\star}, d_{k}\right)=\left(x_{k+c T}^{\star}, u_{k+c T}^{\star}, d_{k+c T}\right)$ holds for any positive integer $c \geq 0$. These conditions allow to apply different EMPC strategies for the control of DWNs.

\section{ECONOMIC MPC STRATEGIES FOR THE MANAGEMENT OF DWNS}

In this section, different EMPC strategies are stated for the DWN control problem in order to be compared. These schemes are: a two-layer architecture where an economic planner and a tracking MPC are interacting, a standard EMPC with terminal state constraint, and two one-layer EMPC strategies that account for changes in the economic criteria.

\section{A. Hierarchical EMPC}

This is a two-layer optimal controller, where a separation of objectives, models and/or time-scales may be performed. Below are stated the optimisation problems involved in this hierarchical approach.

1) Upper layer EMPC: In this layer, an RTO is used to compute at the beginning of each operating cycle the optimal time-varying state and input trajectories using current measurements. The associated FHOP is stated as

$$
\min _{\mathbf{x}_{z}^{r} \mathbf{u}_{z}^{r} \boldsymbol{\xi}_{z}^{r}} \sum_{k=z}^{z+H_{p}^{u}-1} \tilde{V}_{k}\left(x_{k}, u_{k}\right)
$$

subject to (1), (2), (3) $\forall k \in \mathbb{N}_{\left[z, z+H_{p}^{u}-1\right]}$ and

$$
x_{z+H_{p}^{u}}=x_{z}, \quad x_{z}=\bar{x}_{z},
$$

where $H_{p}^{u} \in \mathbb{N}$ is the prediction horizon, $\bar{x}_{z} \in \mathbb{R}^{n_{x}}$ is the measured initial state at time instant $z \in \mathbb{N}$, and $\mathbf{x}_{z}^{r \star} \triangleq\left\{x_{k}^{\star}\right\}_{k=z}^{z+H_{p}^{u}-1}$ and $\mathbf{u}_{z}^{r \star} \triangleq\left\{u_{k}^{\star}\right\}_{k=z}^{z+H_{p}^{u}-1}$ are the optimal state and input trajectories calculated from (7) to govern the lower layer MPC. Defining, $x_{k}^{r} \triangleq x_{k}^{\star}$ and $u_{k}^{r} \triangleq u_{k}^{\star}$ for $k \in \mathbb{N}_{\left[z, z+H_{p}^{u}-1\right]}$, a lower layer MPC is designed as follows.

2) Lower layer Tracking MPC: In this layer, a conventional MPC is used to enforce the system to track the precomputed optimal trajectories. The associated FHOP is stated as

$$
\min _{\mathbf{u}_{t} \boldsymbol{\xi}_{t}} \sum_{k=t}^{t+H_{p}^{l}-1}\left\|x_{k}-x_{k}^{r}\right\|_{Q_{x}}^{2}+\left\|u_{k}-u_{k}^{r}\right\|_{Q_{u}}^{2}
$$

subject to (1), (2), (3) $\forall k \in \mathbb{N}_{\left[t, t+H_{p}^{l}-1\right]}$ and

$$
x_{t+H_{p}^{l}}=x_{t+H_{p}^{l}}^{r}, \quad x_{t}=\bar{x}_{t},
$$

where $H_{p}^{l} \in \mathbb{N}$ is the prediction horizon of the lower layer, $\bar{x}_{t} \in \mathbb{R}^{n_{x}}$ is the measured initial state at time instant $t \in \mathbb{N}$, and $Q_{x}$ and $Q_{u}$ are appropriate positive definite weighting matrices. Following the receding horizon technique, the control law derived under this hierarchical scheme is given by $\kappa\left(\bar{x}_{t}, \mathbf{d}_{t}\right)=u_{t}^{\star}\left(\bar{x}_{t}, \mathbf{d}_{t}\right)$, i.e., only the first control action of the optimal input sequence obtained in (8) is applied to the system. If asymptotic convergence to the upper layer trajectory is desired, the tracking problem can be reformulated in terms of the error $e_{k}=x_{k}-x_{k}^{r}$, leading to a set-point (origin) stabilization problem of the error dynamics; see, e.g., [8].

Note that the prediction horizon $H_{p}^{u}$ of the upper layer must be large enough to cover the nominal operating cycle $T$ and the prediction horizon $H_{p}^{l}$ of the lower layer, i.e., $H_{p}^{u} \geq T+H_{p}^{l}$. Furthermore, the upper layer may have an equal or larger sampling time than the one of the lower layer, i.e., $\Delta t_{1} \geq \Delta t_{2}$. The main drawback of this two-layer MPC approach for the management of DWNs is that if the energy price schedule or the water demand pattern changes in time with a high rate, then the transitory periods will be so that the interaction between layers will lead to a possible loss of feasibility or to an economic performance degradation.

\section{B. Standard EMPC}

The main feature of this approach, in contrast with the hierarchical scheme, is that even when the two-layers may work with the same sampling time, the standard EMPC considers the global cost function directly as the stage cost of the controller objective and avoids penalising the tracking error to the targets. The associated FHOP for the periodic operation of DWNs is stated as

$$
\min _{\mathbf{u}_{t}, \boldsymbol{\xi}_{t}} \sum_{k=t}^{t+H_{p}-1} \tilde{V}_{k}\left(x_{k}, u_{k}\right)
$$

subject to (1), (2), (3) $\forall k \in \mathbb{N}_{\left[t, t+H_{p}-1\right]}$ and

$$
x_{t+H_{p}}=x_{\bmod \left(t+H_{p}, T\right)}^{\star}, \quad x_{t}=\bar{x}_{t},
$$


where $\bar{x}_{t} \in \mathbb{R}^{n_{x}}$ is the measured initial state at time instant $t \in \mathbb{N}$, and $x_{\bmod \left(t+H_{p}, T\right)}^{\star}$ is the optimal periodic value obtained in (6) that corresponds to the time instant $t$. As shown in [1], the standard EMPC is capable of enhancing the economic performance of the system and achieving an asymptotic average cost, which is at least as good as that of the best periodic trajectory. Moreover, convergence can be enforced with the EMPC strategy if the cost function is modified to be dissipative with respect to the optimal periodic trajectory (by adding, e.g., convex regularization terms as done in [1]). The stability of the closed-loop system around the feasible optimal operating cycle can be guaranteed following the results in [20] for periodic systems.

Even when this EMPC controller improves the average economic performance of the DWN, its main weakness is also a possible loss of feasibility, especially if the parameters affecting the cost function, i.e., water and energy prices or priority weights, change the optimal cycle or the target state to unreachable values for the given prediction horizon.

\section{EMPC for DWNs with Changing Operating Patterns}

In order to overcome the possible loss of feasibility due to changing operating patterns caused by the parameters of the cost function or by the demands, two approches following the ideas in [9] and [11] are here proposed to be solved in a one-layer architecture. These schemes integrate in different ways the optimal trajectory problem (6) with the standard EMPC in (9).

\section{1) Option A: Enlargement of the prediction horizon:}

$$
\min _{\mathbf{u}_{t}, \boldsymbol{\xi}_{t}} \sum_{k=t}^{t+H_{p}+T-1} \tilde{V}_{k}\left(x_{k}, u_{k}\right)
$$

subject to (1), (2), (3), and

$$
x_{k+H_{p}+T}=x_{k+H_{p}}, \quad x_{t}=\bar{x}_{t} .
$$

Note that in this option, slight changes of the EMPC framework are required, i.e., the terminal constraint in (10b) is associated to a periodic trajectory that results from the same prediction model used in the optimisation problem rather than to a precomputed trajectory, and the prediction horizon is extended to cover the period of the process dynamics.

2) Option B: Inclusion of a pseudo-reference to track:

$$
\begin{aligned}
\min _{\mathbf{u}_{k} \mathbf{x}_{k}^{s}, \mathbf{u}_{k}^{s}, \boldsymbol{\xi}_{k}} & \sum_{k=t}^{t+H_{p}^{s}-1} \gamma_{\mathrm{O}} \tilde{V}_{k}\left(x_{k}^{s}, u_{k}^{s}\right) \\
& +\sum_{k=t}^{t+H_{p}-1} \gamma_{\mathrm{T}}\left(\left\|x_{k}-x_{k}^{s}\right\|_{Q_{x}}^{2}+\left\|u_{k}-u_{k}^{s}\right\|_{Q_{u}}^{2}\right)
\end{aligned}
$$

subject to (1), (2), (3) $\forall k \in \mathbb{N}_{\left[t, t+H_{p}\right]}$ and

$$
\begin{array}{ll}
x_{k+1}^{s}=A x_{k}^{s}+B u_{k}^{s}+B_{d} d_{t+k}, & \forall k \in \mathbb{N}_{\left[t, t+H_{p}^{s}-1\right]} \\
E_{u} u_{k}^{s}+E_{d} d_{t+k}=0, & \forall k \in \mathbb{N}_{\left[t, t+H_{p}^{s}-1\right]} \\
\epsilon x_{\min } \leq x_{k}^{s} \leq \epsilon x_{\max }, & \forall k \in \mathbb{N}_{\left[t, t+H_{p}^{s}\right]}^{s} \\
\epsilon u_{\min } \leq u_{k}^{s} \leq \epsilon u_{\max }, & \forall k \in \mathbb{N}_{\left[t, t+H_{p}^{s}\right]} \\
x_{k} \geq s-\xi_{k}, \quad \xi_{k} \geq 0, & \forall k \in \mathbb{N}_{\left[t, t+H_{p}^{s}\right]} \\
x_{t+H_{p}^{s}}^{s}=x_{t}^{s}, & \\
x_{t+H_{p}}=x_{t+H_{p}}^{s}, & \\
x_{t}=\bar{x}_{t}, &
\end{array}
$$

where $Q_{x}$ and $Q_{u}$ are positive definite matrices, $\gamma_{\mathrm{O}}$ and $\gamma_{\mathrm{T}}$ are positive scalars introduced to establish a trade-off between economic and tracking performance, and $\epsilon \in(0,1)$ is a tightening factor included to avoid active constraints at the optimal pseudo-reference. The prediction horizons should be selected such that $H_{p}^{s} \geq H_{p}$.

Remark 1: Controllers (10) and (11) are not enforcing convergence to the precomputed optimal trajectory obtained in (6). Instead, they are meant to retain feasibility under possible changes of the economic parameters in the cost function and to find new optimal trajectories for the current conditions. To guarantee asymptotic stability to the trajectory obtained in (6), either when using (10) or (11), the results in [9] and [11] can be applied, respectively, considering the error dynamics (regarding the target periodic trajectory) under the assumptions of controllability of the system and the boundedness and convexity of the constraints and cost function involved.

\section{CASE STUDY}

\section{A. Description}

This section briefly describes a motivational example useful to develop EMPC approaches to solve multi-objective constrained optimisation problems that may appear in the control of DWNs. In general, the DWN operation is driven by the energy prices and the exogenous and endogenous demands. The system under study is a portion extracted from the Barcelona DWN reported in [13]. In Fig. 1, a graphical representation of the DWN example is shown, which contains 2 water sources, 3 tanks, 6 manipulated actuators, 4 demand sectors and 2 intersection nodes.

The matrices and vectors that define the system and constraints are the following:

$$
\begin{aligned}
& A=\left[\begin{array}{lll}
1 & 0 & 0 \\
0 & 1 & 0 \\
0 & 0 & 1
\end{array}\right], \quad B=\left[\begin{array}{llllll}
0 & 0 & 0 & 1 & 1 & 0 \\
0 & 0 & 0 & 0 & 0 & 1 \\
0 & 0 & 1 & 0 & 0 & 0
\end{array}\right] \Delta t, \\
& B_{d}=\left[\begin{array}{cccc}
-1 & 0 & 0 & 0 \\
0 & 0 & -1 & 0 \\
0 & 0 & 0 & -1
\end{array}\right] \Delta t \\
& E=\left[\begin{array}{cccccc}
1 & -1 & -1 & 0 & 0 & -1 \\
0 & 1 & 0 & 0 & -1 & 0
\end{array}\right], E_{d}=\left[\begin{array}{cccc}
0 & 0 & 0 & 0 \\
0 & -1 & 0 & 0
\end{array}\right], \\
& x_{\min }=\left[\begin{array}{lll}
0 & 0 & 0
\end{array}\right]^{\top} \text {, } \\
& x_{\max }=\left[\begin{array}{lll}
470 & 960 & 3100
\end{array}\right]^{\top} \text {, } \\
& u_{\min }=\left[\begin{array}{llllll}
0 & 0 & 0 & 0 & 0 & 0
\end{array}\right]^{\top} \text {, } \\
& u_{\max }=\left[\begin{array}{llllll}
1.2970 & 0.0500 & 0.1200 & 0.0150 & 0.0317 & 0.0220
\end{array}\right]^{\top} \text {. }
\end{aligned}
$$




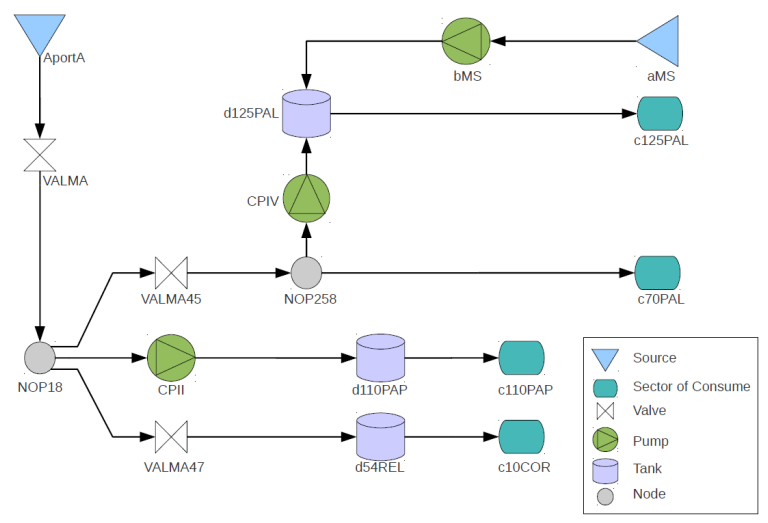

Fig. 1. Topology of the Three-tanks DWN example

\section{B. Results}

This section presents the results of applying the EMPC approaches described in Section III to the aforementioned case study. The sampling time is $\Delta t=3600$ seconds. The simulation horizon is sixteen days ( $N=384$ hours) for each strategy. The weights of the aggregate user-defined cost function are $\lambda_{1}=100, \lambda_{2}=10$, and $\lambda_{3}=0.005$. For the tracking terms, the weighting matrices $\left(Q_{x}\right.$ and $\left.Q_{u}\right)$ are set up as identity matrices of proper dimensions. The prediction horizon has been selected as $H_{p}=24 \mathrm{~h}$, due to the periodicity of both water demands and electricity prices. For the hierarchical controller, the upper layer is executed every $24 \mathrm{~h}$ as usually done in water distribution scheduling, while the lower layer runs in an hourly basis as in the other economic MPC strategies. The initial common state for all simulations is $x_{0}^{\star}=[160.44,646.23,633.89]^{\top}$ in $\mathrm{m}^{3}$ and the security threshold is $s=[42,18.0,270]^{\top}$ in $\mathrm{m}^{3}$. The simulations have been carried out using CPLEX 12.5 and Matlab $^{\circledR}$ R2010b (64 bits), running in a PC Intel ${ }^{\circledR}$ Core $^{\text {TM }}$ E8600 at $3.33 \mathrm{GHz}$ with $8 \mathrm{~GB}$ of RAM. The closed-loop performance of each controller has been assessed using the following average key performance indicators (KPI) for the economic, safety and smoothness objectives, respectively:

$$
\begin{aligned}
& \mathrm{KPI}_{E} \triangleq \frac{1}{N} \sum_{k=1}^{N}\left(\alpha_{1}+\alpha_{2, k}\right)^{\top}\left|\mathbf{u}_{k}\right| \Delta t, \\
& \mathrm{KPI}_{S} \triangleq \frac{1}{N} \sum_{k=1}^{N} \sum_{i=1}^{n_{x}} \xi_{(i), k}, \\
& \mathrm{KPI}_{\Delta U} \triangleq \frac{1}{N} \sum_{k=1}^{N}\left\|\Delta u_{k}\right\|^{2} .
\end{aligned}
$$

Results are summarised in Table I. The safety indicator has been omitted in this table given that, for all simulated scenarios and strategies, $\mathrm{KPI}_{S}=0$, which means that all of the MPC controllers decided not to use water from the safety-stocks for the given periodic demand. Note that for each strategy the enforcement of terminal constraints implies an increment of the economic cost. This decrease in
TABLE I

COMPARISON OF CONTROLLER PERFORMANCE

\begin{tabular}{lcc}
\hline Controller & KPI $_{E}$ & KPI $_{\Delta U}$ \\
\hline Ideal scheduling (Problem (6)) & 28.6056 & $2.06 \times 10^{-3}$ \\
\hline HEMPC $_{(1)}$ & 28.4347 & $2.14 \times 10^{-3}$ \\
\hline HEMPC $_{(2)}$ & 28.6114 & $2.08 \times 10^{-3}$ \\
\hline EMPC $_{(1)}$ & 28.4124 & $8.89 \times 10^{-7}$ \\
\hline EMPC $_{(2)}$ & 28.6080 & $8.89 \times 10^{-7}$ \\
\hline EMPCT-A $_{(1)}$ & 28.4124 & $8.89 \times 10^{-7}$ \\
\hline EMPCT-A $_{(2)}$ & 28.6041 & $8.89 \times 10^{-7}$ \\
\hline EMPCT-B $_{(1) @\left\{\gamma_{\mathrm{O}}=1, \gamma_{\mathrm{T}}=1\right\}}$ & 28.5165 & $2.17 \times 10^{-3}$ \\
\hline EMPCT-B $_{(1) @\left\{\gamma_{\mathrm{O}}=1, \gamma_{\mathrm{T}}=10\right\}}$ & 28.4493 & $2.35 \times 10^{-3}$ \\
\hline EMPCT-B $_{(1) @\left\{\gamma_{\mathrm{O}}=1, \gamma_{\mathrm{T}}=100\right\}}$ & 28.4178 & $2.34 \times 10^{-3}$ \\
\hline EMPCT-B $_{(1) @\left\{\gamma_{\mathrm{O}}=10, \gamma_{\mathrm{T}}=1\right\}}$ & 28.8128 & $2.29 \times 10^{-3}$ \\
\hline EMPCT-B $_{(2) @\left\{\gamma_{\mathrm{O}}=1, \gamma_{\mathrm{T}}=100\right\}}$ & 28.6120 & $2.43 \times 10^{-3}$ \\
\hline
\end{tabular}

Subindex (1) indicates non-periodic behaviour enforced while (2) indicates that the periodic constraint is enforced.

\begin{tabular}{|c|c|c|c|}
\hline Controller & $\begin{array}{l}\text { Water Cost } \\
\text { (e.u./day) }\end{array}$ & $\begin{array}{l}\text { Electric Cost } \\
\text { (e.u./day) }\end{array}$ & $\begin{array}{l}\text { Daily Cost } \\
\text { (e.u./day) }\end{array}$ \\
\hline $\mathrm{EMPC}_{(2)}$ & 577.24 & 110.04 & 687.28 \\
\hline $\operatorname{HEMPC}_{(2)}$ & 610.02 & 134.13 & 744.15 \\
\hline EMPCT-A $_{(2)}$ & 577.79 & 109.56 & 687.35 \\
\hline EMPCT-B $_{(2)}$ & 577.75 & 109.79 & 687.54 \\
\hline
\end{tabular}

TABLE II

COMPARISON OF DAILY AVERAGE COSTS OF EMPC STRATEGIES

performance is the price for gaining in stability. Furthermore, Table II discloses details of the production and operational costs related to each strategy starting from a non-optimal state $x_{0}=[92.45,905.82,504.14]^{\top}$ in $\mathrm{m}^{3}$, and compares the daily average economic performance $\left(\mathrm{DAP}=24 \times \mathrm{KPI}_{E}\right.$ ) of the controllers enforcing their corresponding periodic terminal constraints. For the standard $\mathrm{EMPC}_{(2)}$, the terminal constraint is set up in relation to a pre-calculated optimal cycle obtained from (6). In the $\operatorname{HEMPC}_{(2)}$, the reference trajectory is computed by the upper layer every $24 \mathrm{~h}$. For controllers EMPCT-A E2) and EMPCT-B $(2)$, no pre-calculated trajectory is needed. It can be seen how the $\operatorname{HEMPC}_{(2)}$ cost degrades notoriously the performance in comparison with the other MPC strategies due to the time-scale separation in its layers. Even when feasibility issues were not found for any of the strategies in this case study, these results reaffirm the current tendency of improving the economic performance by migrating to one-layer EMPC which are robust to changes in the cost function.

In order to further highlight the performance of the EMPC controllers described in Section III-C that copes with chang- 


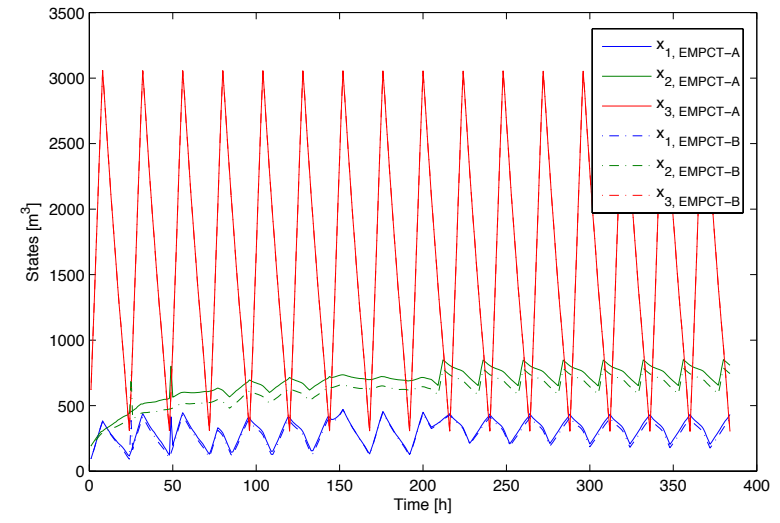

Fig. 2. Evolution of some states under varying economic parameters
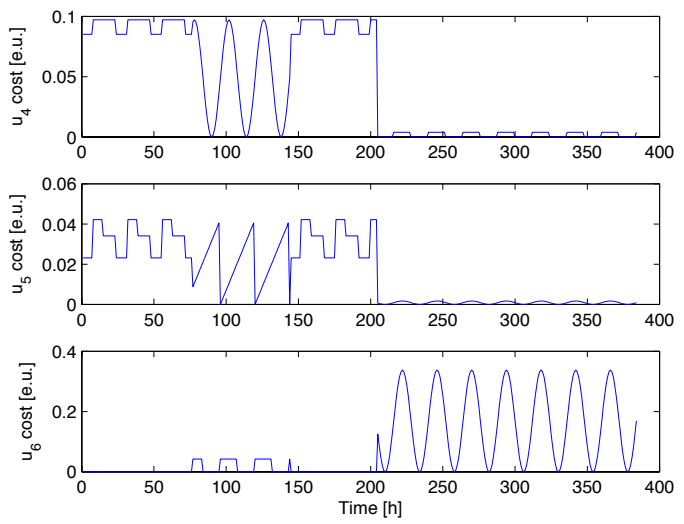

Fig. 3. Price-of-use for actuators $u_{4}, u_{5}$ and $u_{6}$ in economic units (e.u)

ing economic criterion, the price parameter of the economic term in (4) has been affected switching the price profile at different time instants but keeping the same period, see Fig. 3. As it can be seen in Fig. 2 for the evolution of the states, both controllers maintain the recursive feasibility and stabilise at similar trajectories. Even so, the approach in (11), which includes a pseudo reference and tracking terms, presents a slightly higher cost with respect to the approach in (10). This behaviour might be due to the regularisation terms that decrease the economic performance if design parameters are not properly tuned.

\section{CONCLUSIONS}

In this paper, the potential of economic MPC for the management of DWNs has been verified on a proof of concept case study. A multi-objective cost function was considered and different EMPC formulations were analysed and extended for controlling a supply water nominal system, where water demands were considered periodic and perfectly known. Especially, the single-layer EMPC approaches resulted to be of great utility for the control of DWNs due to their capacity to cope with changes in the economic parameters of the cost function. Future research lines are the stochastic EMPC formulations to enhance robustness with the minimum performance degradation, the tuning of the EMPC that affects the DWN performance, and the extension of the feasibility, stability and convergence results when dealing with large-scale systems decomposed for decentralised or distributed control.

\section{REFERENCES}

[1] D. Angeli, R. Amrit, and J.B. Rawlings. On average performance and stability of economic model predictive control. Automatic Control, IEEE Transactions on, 57(7):1615-1626, 2012.

[2] C. Biscos, M. Mulholland, M.V. Le Lann, C.A. Buckley, and C.J. Brouckaert. Optimal operation of water distribution networks by predictive control using MINLP. Water SA, 29:393-404, 2003.

[3] J. Burgschweiger, B. Gnädig, and M.C. Steinbach. Optimization models for operative planning in drinking water networks. Optimization and Engineering, 10(1):43-73, 2009.

[4] A. Castelletti, F. Pianosi, and R. Soncini-Sessa. Stochastic and robust control of water resource systems: Concepts, methods and applications. In System Identification, Environmental Modelling, and Control System Design, pages 383-401. Springer London, 2012.

[5] G. Cembrano, G. Wells, J. Quevedo, R. Pérez, and R. Argelaguet. Optimal control of a water distribution network in a supervisory control system. Control Engineering Practice, 8(10):1177-1188, 2000.

[6] M. Ellis and P.D. Christofides. Integrating dynamic economic optimization and model predictive control for optimal operation of nonlinear process systems. Control Engineering Practice, 22(0):242251, 2014.

[7] S. Engell. Feedback control for optimal process operation. Journal of Process Control, 17(3):203 - 219, 2007.

[8] T. Faulwasser and R. Findeisen. A model predictive control approach to trajectory tracking problems via time-varying level sets of Lyapunov functions. In Proc. 50th IEEE Conference on Decision and Control and European Control Conference (CDC-ECC), pages 3381-3386, Orlando, FL, USA, December 2011.

[9] R. Huang, E. Harinath, and L.T. Biegler. Lyapunov stability of economically oriented NMPC for cyclic processes. Journal of Process Control, 21(4):501-509, 2011

[10] M. Kansal, G. Arora, C. León, S. Martín, J. Elena, and J. Luque. Explore-hybrid expert system for water networks management. Journal of Water Resources Planning and Management, 127(6):415-416, 2001

[11] D. Limon, M. Pereira, D. Muñoz de la Peña, T. Alamo, and J.M Grosso. Single-layer economic model predictive control for periodic operation. Journal of Process Control, 2014. (Accepted for publication).

[12] J.M. Maciejowski. Predictive control with constraints. Prentice Hall, Essex, England, 2002.

[13] C. Ocampo-Martinez, V. Puig, G. Cembrano, and J. Quevedo. Application of predictive control strategies to the management of complex networks in the urban water cycle. Control Systems, IEEE, 33(1):1541, 2013.

[14] J. Pascual, J. Romera, V. Puig, R. Creus, and M. Minoves. Operational predictive optimal control of Barcelona water transport network. In Proc. 18th IFAC World Congress, pages 10571-10578, Milano, Italy, August 2011.

[15] J.B. Rawlings, D. Angeli, and C.N. Bates. Fundamentals of economic model predictive control. In Proc. 51st IEEE Conference on Decision and Control $(C D C)$, pages 3851-3861, 2012.

[16] A. Shepherd and L. Ortolano. Water-supply system operations: Critiquing expert-system approach. Journal of Water Resources Planning and Management, 122(5):348-355, 1996.

[17] P. Tatjewski. Advanced control and on-line process optimization in multilayer structures. Annual Reviews in Control, 32(1):71 - 85, 2008.

[18] J. Vieira, M.C. Cunha, L. Nunes, J.P. Monteiro, L. Ribeiro, T. Stigter, J. Nascimento, and H. Lucas. Optimization of the operation of largescale multisource water-supply systems. Journal of Water Resources Planning and Management, 137(2):150-161, 2011.

[19] L. Würth, R. Hannemann, and W. Marquardt. A two-layer architecture for economically optimal process control and operation. Journal of Process Control, 21(3):311-321, 2011.

[20] M. Zanon, S. Gros, and M. Diehl. A Lyapunov function for periodic economic optimizing model predictive control. In Proc. 52nd IEEE Annual Conference on Decision and Control (CDC), pages 5107-5112, Firenze, Italy, December 2013. 